\title{
PET/CT imaging analysis of recurrent sites and patterns of spread following modified radical surgery (type B) for stage Ib-IIa cervical cancer
}

\author{
SHUXIA CHENG, YANMEI LI, MINGCHUAN ZHANG and TINGTING TONG \\ Department of Gynecologic Oncology, Affiliated Cancer Hospital of Zhengzhou University, \\ Zhengzhou, Henan 450003, P.R. China
}

Received May 13, 2017; Accepted November 21, 2017

DOI: $10.3892 / \mathrm{ol} .2018 .9070$

\begin{abstract}
The aim of the present study was to use imaging to retrospectively analyze the recurrent sites and patterns of spread of early cervical cancer following radical surgery. A total of 86 patients with cervical cancer (stage IB or IIA) showed postoperative pelvic recurrences and distant metastases. Based on positron emission tomography or computed tomography, the correlation between clinicopathologic factors and sites of recurrence was determined and the pattern of spread was evaluated. Among the 86 patients with postoperative recurrence of early cervical cancer, 76 exhibited pelvic recurrence involving the cardinal and uterosacral ligaments. Other recurrences were paravaginal $(n=14)$ and vaginal $(n=10)$. Seven cases of recurrence were in the pelvic lymph nodes and 10 patients had distant metastases. The rate of paracervical ligament recurrence in patients with stromal invasion $\geq 1 / 2$ depth was higher, compared with that in patients with stromal invasion $<1 / 2$ depth, however, the difference was not statistically significant. The rate of distant metastases in patients with positive pelvic lymph nodes was higher, compared with that in patients with negative lymph nodes $(\mathrm{P}=0.001)$. Recurrent lesions spread along the residual ligament to the lateral pelvic wall. It was found that, following radical surgery for early cervical cancer, recurrent tumor spread was predominantly confined to the subperitoneal residual ligaments of the cervix and vagina. For patients with positive lymph nodes, the incidence of distant metastases increased significantly.
\end{abstract}

\section{Introduction}

Cervical cancer is one of the major types of malignant tumor that has a negative impact on women's health. In economically

Correspondence to: Dr Shuxia Cheng, Department of Gynecologic Oncology, Affiliated Cancer Hospital of Zhengzhou University, 127 Dongming Road, Jinshui, Zhengzhou, Henan 450003, P.R. China E-mail: qianpengcheng56@sina.com

Key words: positron emission tomography/computed tomography, cervical cancer, cancer recurrence, patterns of spread underdeveloped countries, cervical cancer is the leading cause of mortality among women (1). According to an epidemiologic study, the incidence of cervical cancer and associated mortality rates in China have increased yearly over the last 10 years (2). In addition, due to the implementation of cervical cancer screening, the detection of early cervical cancer has increased; however, the recurrence rate remains at $\sim 30 \%$. Post-operative recurrences are correlated to clinical stage, surgical method and post-operative pathologic reports (3). However, the correlation between surgical method and prognosis is weaker. In 2015, the National Comprehensive Cancer Network (NCCN) guidelines for the clinical diagnosis and treatment of cervical cancer updated the criteria for cervical cancer surgery. The new guidelines recommend using the Querleu and Morrow (QM) surgical classification system (4). In China, modified radical hysterectomy is a common surgical procedure for cervical cancer, which is similar to the B type of the QM classification (5). In the present study, the location of recurrence and the route of metastasis in 86 patients with early stage cervical cancer were retrospectively analyzed following a type B surgical procedure. The present study aimed to retroactively analyze the recurrent sites and patterns of spread following radical surgery for early cervical cancer. In doing so, the associations between recurrence and metastasis, age, time, and postoperative pathologic changes were investigated.

\section{Materials and methods}

Clinical data. The present study was a single site retrospective study performed between October 2009 and March 2015 involving 86 patients who were diagnosed with cervical cancer metastases and recurrence following surgery at Henan Cancer Hospital (Henan, China). The patient ages ranged between 31 and 79 years, with a median age of 45 years, and all patients had complete medical records. Of the 86 patients, 78 had squamous cell carcinoma of the uterine cervix and 8 had adenocarcinoma. The primary treatment was modified radical hysterectomy with bilateral pelvic lymph node dissection. With respect to the classification criteria for surgery, according to the 2015 NCCN clinical practice guidelines for cervical cancer, all of the patients were classified as having cervical cancer radical surgery type $B$. The post-operative pathologic 
diagnosis was confirmed and all surgical margins were negative. There was no post-operative residual tumor tissue. None of the patients received radiotherapy prior to the recurrence (Table I).

Positron emission tomography/computed tomography (PET/CT) (General Electric Company, New Fairfield, Connecticut, American). When a recurrence or metastasis was suspected in patients with early stage cervical cancer, they underwent a whole-body PET/CT examination. All local recurrences were pathologically confirmed. If a distant metastasis biopsy was difficult to obtain, further confirmation was required. In the present study, all of the patients received at least one PET/CT examination.

Statistical analysis. SPSS 17.0 statistical software (SPSS, Inc., Chicago, IL, USA) was used for analyses. $\chi^{2}$ and Fisher's exact probability tests were used to compare the constitute ratios. $\mathrm{P}<0.05$ was considered to indicate a statistically significant difference.

\section{Results}

Association between recurrence and metastasis, age, time of recurrence and post-operative pathologic changes. Among the sites of post-operative recurrence and metastases, 45 simple recurrences involved the primary sacral ligament, 10 were vaginal recurrences, 14 were paravaginal recurrences, seven involved the pelvic lymph nodes, and five were pelvic metastases. Metastasis to the sacral ligament and pelvic region occurred in three patients and simple pelvic metastases occurred in two patients (Table I). The rate of recurrence was $79 \%(68 / 86)$ with a pelvic metastatic rate of $100 \%(10 / 10)$ within 2 years following surgery.

In patients with negative margins, negative lymph nodes, a cervical stromal invasion depth of $<1 / 2$ and a cervical stromal invasion depth of $>1 / 2$, the cancer recurrence rates at the primary sacral ligament remnant and the vagina were $40 \%$ (4/10), $10 \%$ (1/10), $40 \%$ (4/10), $60.3 \%$ (35/58), $20.7 \%(12 / 58)$ and $10.3 \%(6 / 58)$, respectively.

With the increase in the depth of invasion, the recurrence rate of cancer of the uterine cervix was greater than the vaginal recurrence rate $\left(\chi^{2}=6.24, \mathrm{P}=0.031\right)$. The incidence of pelvic lymph node and distant metastases was $50 \%(5 / 10)$, which was higher when compared with the pelvic lymph node-negative patients [7\% (5/76); $\mathrm{P}=0.001]$. The median relapse time was 8 months, which was significantly shortened.

In patients $>60$ years of age, the paravaginal recurrence rate was significantly higher, compared with that in the $<60$ years of age group $\left(\chi^{2}=19.9, \mathrm{P}<0.01\right)$. With increasing age, the recurrence time was prolonged (Table II). In terms of metastases, three patients were identified with pulmonary metastases, five with abdominal aortic lymph node metastases, one with small intestine metastasis and one patient with greater retinal metastasis.

\section{Transfer pathway}

Cancer recurrence and metastasis along the main ligament. Among the 85 patients in included the present study, cancer spread along the cardinal ligament in 27 patients, including
19 first diagnosed with a proximal cardinal ligament stump and eight with infiltration along the total length of the remaining ligament all the way to the pelvic wall. Continuous images in combination with a change in the standardized uptake value $(\mathrm{SUV})=$ showed that the cardinal ligament invasion pathways were as follows: The earliest cancer recurrence involved the cardinal ligament stump, followed by the internal iliac vascular axis (the main vascular ligament) as the center, followed by a spread outwards and upwards, close to the iliac arterial bifurcation and terminating at the basin wall between the internal and external iliac vessels (Fig. 1A-D).

Invasion and metastatic recurrence of cervical cancer along the uterosacral ligament. In the present study, the recurrence and metastasis along the sacral ligament occurred in 21 patients, 18 of whom were first diagnosed with involvement of the proximal sacral ligament stump. Infiltration of the entire length of the residual sacral ligament occurred in three patients, reaching the third and fourth vertebral bodies. Continuous images in combination with changes in the SUV showed that the invasion pathway along the uterosacral ligament was recurrent tumor infiltration from the sacral ligament stump. The cancer spread to the mesorectum and backward to the second, third and fourth sacral vertebrae. If the invasion continued to expand, the cancer invaded the mesorectum, the piriformis muscle and the soft tissues of the pelvic wall, as shown in Fig. 2A-D.

Tumor recurrences spread along the vagina and surrounding tissue ligaments. In the present study, 11 patients had vaginal recurrences and seven patients had an abnormal vaginal discharge. Regular examination via Thinprep cytologic test vaginal cytology revealed abnormalities in eight cases. Biopsies of the vaginal mucosal surfaces showed that the tumors or vaginal abnormalities were squamous cell carcinomas. Paravaginal recurrences were found in 13 patients. Deep myometrial invasion was demonstrated in post-operative pathology. If the patient had no abnormal vaginal discharge, abnormal cytology or abnormal findings on gynecologic examination of the vaginal mucosal, a submucosal paravaginal tissue mass was noted and a biopsy was performed. Paravaginal tumors arose in the fascial tissues, of which nine cases involved the cervical vaginal ligament, which spread forwards and medially to invade the posterior walls of the ureter and bladder, and then infiltrated downwards to the urethra. The tumor spread laterally and posteriorly along the same side of the rectovaginal fascia tumor, as shown in Fig. 3A-D. In the present study, five patients had vaginal and rectal fascia or vaginal fascia involvement, however, no recurrences were noted in the anterior pelvic wall space.

\section{Discussion}

Clinical studies $(6,7)$ have shown that the recurrence and metastasis rate of stage IB-IIA cervical cancer is $\sim 30 \%$. The majority of the metastatic tumors were pelvic metastases. With an increase in risk factors, including lymph node metastasis, infiltration of the uterus and tumor diameter $\geq 2 \mathrm{~cm}$, the post-operative recurrence rate can increase to $40-50 \%$. Post-operative concurrent radiotherapy and chemotherapy can reduce the recurrence rate to $46 \%$, however, there are $15 \%$ of patients remaining, who exhibit post-operative radiotherapy recurrence. Distant 
Table I. Association between metastatic site and time and post-operative pathologic changes.

\begin{tabular}{|c|c|c|c|c|c|c|c|}
\hline \multirow[b]{2}{*}{$\begin{array}{l}\text { Post-operative } \\
\text { pathology }\end{array}$} & \multirow[b]{2}{*}{ Cases (n) } & \multirow[b]{2}{*}{$\begin{array}{l}\text { Median } \\
\text { recurrence } \\
\text { (months) }\end{array}$} & \multicolumn{5}{|c|}{ Pelvic recurrence site (cases) } \\
\hline & & & $\begin{array}{c}\text { Primary } \\
\text { cardinal and } \\
\text { sacral ligament }\end{array}$ & Vagina & Paravaginal & $\begin{array}{l}\text { Pelvic } \\
\text { lymph } \\
\text { nodes }\end{array}$ & $\begin{array}{c}\text { Only } \\
\text { external Pelvic } \\
\text { metastases (cases) }\end{array}$ \\
\hline $\mathrm{SCC}<1 / 2$ full-thickness invasion & 10 & 24 & 4 & 4 & 1 & 1 & 0 \\
\hline $\mathrm{SCC} \geq 1 / 2$ full-thickness invasion & 58 & 22 & 35 & 6 & 12 & 4 & 1 \\
\hline Adenocarcinoma & 8 & 21 & 6 & - & - & 2 & 0 \\
\hline Lymph node metastasis and other & 10 & 8 & 3 & - & 1 & 5 & 1 \\
\hline
\end{tabular}

SCC, squamous cell carcinoma.

Table II. Association between metastatic site and age.

\begin{tabular}{|c|c|c|c|c|c|c|}
\hline \multirow[b]{2}{*}{$\begin{array}{l}\text { Age } \\
\text { (years) }\end{array}$} & \multirow[b]{2}{*}{ Cases (n) } & \multirow[b]{2}{*}{$\begin{array}{l}\text { Median } \\
\text { recurrence (months) }\end{array}$} & \multicolumn{3}{|c|}{ Pelvic recurrence site (cases) } & \multirow[b]{2}{*}{$\begin{array}{c}\text { External pelvic } \\
\text { metastases (cases) }\end{array}$} \\
\hline & & & $\begin{array}{c}\text { Primary } \\
\text { sacral ligament }\end{array}$ & $\begin{array}{l}\text { Vaginal and } \\
\text { paravaginal }\end{array}$ & $\begin{array}{c}\text { Pelvic } \\
\text { lymph nodes }\end{array}$ & \\
\hline $30-40$ & 16 & 14.2 & 8 & 3 & 4 & 1 \\
\hline $41-60$ & 51 & 18.1 & 35 & 8 & 7 & 1 \\
\hline $61-70$ & 19 & 21.3 & 5 & 13 & 1 & 0 \\
\hline
\end{tabular}
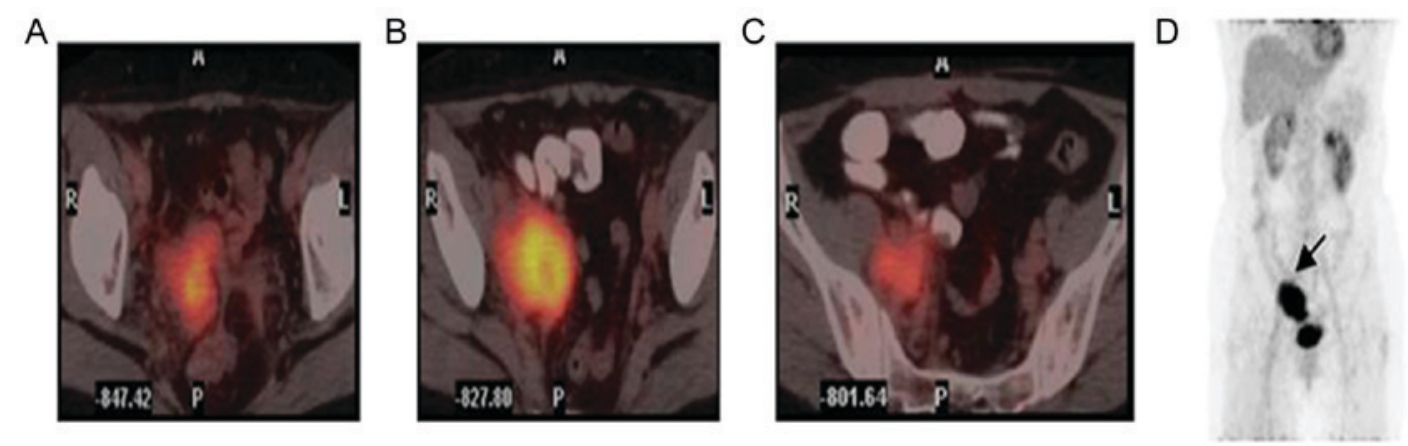

Figure 1. Post-operative recurrence of cervical cancer and spread along the cardinal ligament from the remnant to the pelvic wall. (A) PET/CT cross-sectional images showing that the cancer was located at the right cardinal ligament stump. (B) PET/CT cross-sectional images demonstrating tumor spread along the vessel of the main ligament to the upper and lateral walls of the basin. (C) PET/CT imaging showing tumor close to the the iliac arterial bifurcation spreading to the pelvic wall. (D) PET image (coronal view) where the arrow indicates cancer recurrence spreading from the remnant to the oblique lateral wall of the basin and finally to the common iliac vessels. PET/CT, positron emission tomography/computed tomography.

metastasis is the main contributor to mortality rates in patients with cervical cancer recurrence (8). Stratified studies on the mode of surgery have been limited, and the precise location of pelvic recurrences, and the examination of invasion and spread are also deficient. Gene mutations, metabolic abnormalities and morphological changes are essential in the process of tumor development. PET/CT combined with imaging medicine and tumor metabolism can be used for follow-up monitoring of cervical cancer following treatment (9), which not only facilitates earlier detection of recurrence and metastatic lesions (10), but also assists in confirming the metastasis of local cancer. In the present study, PET/CT was used to analyze the first site of recurrence of cervical cancer, and the anatomical basis of invasion and spread.
Post-operative recurrence and metastasis in type B radical resection in the 86 patients with early cervical cancer showed that the simple parametrial recurrence rate was $67.4 \%(58 / 86)$, the vaginal recurrence rate was $12.8 \%(11 / 86)$, the pelvic lymph node recurrence rate was $8.1 \%$ (7/86), the pelvic lymph node and extra-pelvic metastatic rate was $5.8 \%(5 / 86)$, the parametrial recurrence and pelvic metastatic rate was $3.5 \%$ (3/86) and the simple pelvic external transfer rate was $2.3 \%$ (cultured). The results were similar to the results reported by Wang et al (11). Among the 86 patients, 45 had simple uterine cervix recurrences, including recurrences of the primary sacral ligament and 13 vaginal recurrences, which is the first site of recurrence. For patients with negative margins, negative lymph nodes and an interstitial infiltration depth $\geq 1 / 2$, the recurrence 

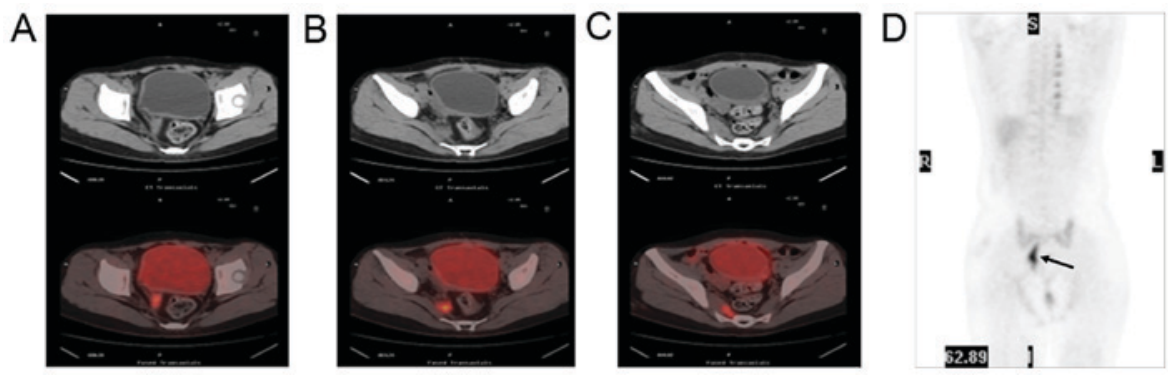

Figure 2. Post-operative recurrence of cervical cancer along the uterosacral ligament from the remnant to the pelvic wall. (A) PET/CT cross-sectional images with right sacral ligament stump cancer. (B) PET/CT cross-sectional images of the right middle sacral ligament tumor. (C) PET/CT cross-sectional images showing the paravertebral tumor located in the right sacral ligament close to the fourth sacral vertebrae. The morphology did not alter significantly. (D) PET/CT images; the arrow indicates the spread of cancer to the mesorectum, and backwards to the second, third and fourth sacral vertebrae. PET/CT, positron emission tomography/computed tomography.
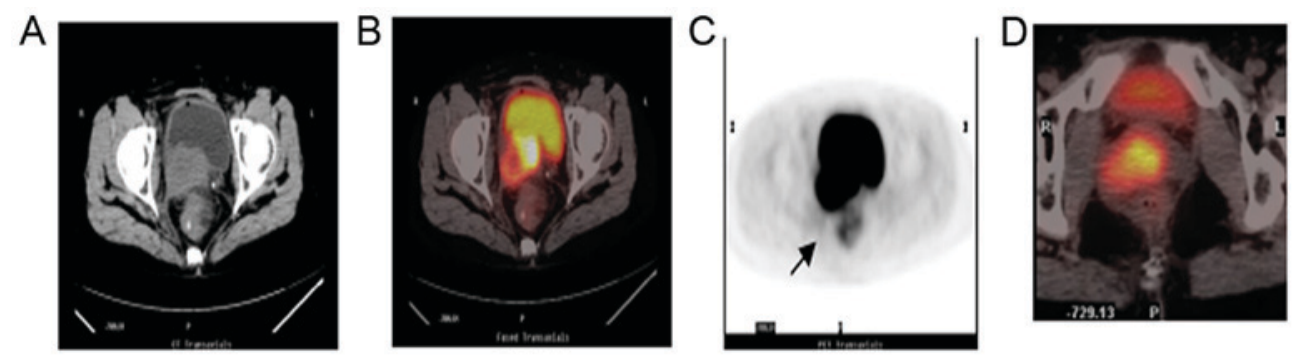

Figure 3. Tumor recurrence and spread along the vagina and surrounding ligaments. The vaginal mucosa was smooth and the submucosal mass was on the right side of the vaginal wall. (A) CT cross-sectional images showing right posterior vaginal stump mass with infiltration of the ureter. (B) PET/CT cross-sectional images of the same layer, on the right side of the bladder vaginal ligament, demonstrating tumor spread along the ipsilateral rectovaginal fascia. (C) PET image of the same layer; the arrow indicates paravaginal cancer. (D) PET/CT cross-sectional images demonstrating the cancer spread downwards along the right side of the rectovaginal fascia; the tissue morphology was not altered significantly. PET/CT, positron emission tomography/computed tomography.

rate was higher, compared with patients with interstitial infiltration $<1 / 2$ depth. The parametrial and vaginal recurrence rates were reduced $\left(\chi^{2}=6.24, \mathrm{P}=0.031\right)$. Elit et al (12) summarized and analyzed 17 retrospective clinical studies and found that the detection rate for recurrences of cervical cancer following surgery were as follows: Physical examination, 29-71\%; CT, $0-34 \%$; vaginal cytology, $0-17 \%$. As the principal method of screening for cervical cancer, vaginal cytology is significant in reducing the incidence of cervical cancer, but is less important in detecting recurrent cervical cancer. The present study showed that the recurrence of early invasive cervical cancer was primarily extended from the mid-uterus to the vagina. The tumor continues to grow and infiltrates the surrounding tissue, including the vagina. Prior to positive-vaginal cytology, the tumor has formed and can be detected by gynecologic examination and imaging. Therefore, the monitoring of post-operative patients, imaging and physical examinations are more useful, compared with vaginal cytology. In addition, the present study showed the correlation between age and site of recurrence; for elderly patients, particularly patients $>60$ years of age, the recurrence rate was $68.4 \%$ (13/19) in the vaginal and paravaginal tissues. Compared with the $\leq 60$-year-old group, in which the recurrence rate was $16.4 \%$ (11/67), the recurrence rate was statistically increased in the $\geq 60$-year-old group $\left(\chi^{2}=19.9, \mathrm{P}<0.01\right)$.

Landoni et al (13) pathologically analyzed the tissue specimens of 230 patients with cervical cancer stage IB-IIA, following radical surgery. The proportion of cancer infiltration to the main ligaments (vesicouterine and uterosacral) and rectovaginal septum were $34,23,15$ and $15 \%$, respectively. The paracervical infiltration depth was first associated with cervical stromal infiltration. The post-operative cervical cancer recurrence can spread along the lateral residual sacral and vaginal ligaments to the pelvic wall. According to the characteristics of $\mathrm{PET} / \mathrm{CT}$ images in several patients, the invasion pathway of the main ligament was as follows: Continuous images in combination with changes in SUV demonstrated that cardinal ligament invasion pathways were the earliest cancer recurrence in the cardinal ligament stump. The internal iliac vascular axis (the main vascular ligament) was central with cancer spreading outwards and upwards, close to the iliac arterial bifurcation, terminating at the basin wall between the internal and external iliac vessels. Recurrent tumor infiltration occurred from the sacral ligament stump, and extended from the mesorectum backwards to the second, third and fourth sacral vertebrae. If invasion continued, the cancer invaded the mesorectum, the piriformis muscle and soft tissues of the pelvic wall, as shown in Fig. 2. If the patient had no abnormal vaginal discharge, abnormal cytology and the gynecologic examination of the vaginal mucosal was normal, but a submucosal paravaginal tissue mass was palpated, a biopsy was performed. Paravaginal tumors arose in the fascial tissues in nine cases, which involved the cervical vaginal ligament and progressed to the medial aspect of the posterior wall of the ureter and bladder, further infiltrating downward to the urethra. The tumor spread along the same side of the rectovaginal fascia, as shown in Fig. 3, in agreement with Xiaohong et al and Zhou et al $(14,15)$, who found that the majority of the sacral ligaments attached to the 
first, second and third sacral vertebrae, and the morphology of the fourth vertebral body was altered. The recurrence rate of cervical deep muscle invasive carcinoma was twice that of vaginal recurrences. The PET/CT imaging showed that the tumor appeared in the vaginal stump and vaginal bladder ligament. The primary sacral ligament is a three-dimensional connective tissue extending from the cervix to the upper vagina. The primary sacral ligament exists as a complex form in the cervix and vagina, separates in the process of extending outward, and terminates on the side wall of the basin and the sacrum, whereas the main sacral ligament complex extends down to the vagina. Following surgery, the remnants spread out through the residual ligament, and the type and modality of surgery affects the rate of recurrence of early cervical cancer $(16,17)$. Following a type B radical resection, the basin wall of each ligament remains partially connected. The tumor can spread along the main and paravaginal ligaments, and invade the adjacent organs.

In the early stage, the local spread of cervical cancer is not random. Höckel (18) and Höckel et al (19) reported that early cervical cancer invasion and metastasis are limited to the cervix, uterus, and the surrounding mesentery ligaments. These tissues are derived from the gyneduct in the embryonic stage. For tissues from other embryonic sources, there was less infiltration, even for adjacent structures, including nervous tissue. For patients with cervical cancer in early stage IB-IIA, total hysterectomy (removal of the pelvic wall of the main sacral ligament) can preserve the nerves and avoid post-operative prophylactic radiotherapy. In the present study, $67.4 \%$ of the patients with recurrent cervical cancer had tumors confined to the posterior peritoneum with spread along these ligaments. These ligaments are also barriers to outward spread of the tumor. In the early stage, recurrence along the ligament is rare and metastasis of the pelvic peritoneal organs is singular. In the present study, only one patient with squamous cell carcinoma of the vagina had intestinal obstruction and one patient with adenocarcinoma had metastasis to the abdominal cavity.

The biological behavior of tumor development determines its treatment. The present study analyzed the recurrence and spread of early stage cervical cancer following type B radical surgery, and the results showed that the recurrence rateinthe cardinal and uterosacral ligament was $52.3 \%$, and that thiswas the most common site of recurrence. According to the QM classification, C type surgery is always recommended in cervical cancer stage IB-IIA. Type C1 surgery is recommended to maintain the sacral ligament close to the pelvic wall of the resection and preservation of the bladder nerve. For patients with adjuvant radiotherapy following type B surgery, all cervical residual ligaments require treatment as part of the region involved, however, the dosage of radiation requires further evaluation. As the patients in the present study were from a number of treatment centers, heterogeneity cannot be excluded. Therefore, to improve the treatment of cervical cancer, a prospective clinical study is required.

\section{Acknowledgements}

This study was supported by the Science and Technology Department of Henan province (grant no. 132300410047).

\section{Competing interests}

The authors declare that they have no competing interests.

\section{References}

1. Jemal A, Bray F, Center MM, Ferlay J, Ward E and Forman D: Global cancer statistics. CA Cancer J Clin 61: 69-90, 2011.

2. Hu SY, Zheng RS, Zhao FH, Zhang SW, Chen WQ and Qiao YL: Trend analysis of cervical cancer incidence and mortality trends from 1989 to 2008 in Chinese women. Zhongguo Yi Xue Ke Xue Yuan Xue Bao 36: 119-125, 2014 (In Chinese).

3. Ryu SY, Kim MH, Nam BH, Lee TS, Song ES, Park CY, Kim JW, Kim YB, Ryu HS, Park SY, et al: Intermediate-risk grouping of cervical cancer patients treated with radical hysterectomy: A Korean Gynecologic Oncology Group study. Br J Cancer 110: 278-285, 2014.

4. Querleu D and Morrow PC: Classification of radical hysterectomy. Lancet Oncol 9: 297-303, 2008.

5. Khatun S, Huda AQ, Begum SK and Ferdous J: Evaluation of pelvic lymphadenectomy during radical hysterectomy for cervical cancer. Mymensingh Med J 26: 287-292, 2017.

6. Rotman M, Sedlis A, Piedmonte MR, Bundy B, Lentz SS, Muderspach LI and Zaino RJ: A phase III randomized trial of postoperativepelvic irradiation in Stage IB cervical carcinoma with poor prognostic features: Follow-up of a gynecologic oncology group study. Int J Radiat Oncol Biol Phys 65: 169-176, 2006.

7. Monk BJ, Wang J, Im S, Stock RJ, Peters WA III, Liu PY, Barrett RJ II, Berek JS, Souhami L, Grigsby PW, et al: Rethinking the use of radiation and chemotherapy afterradical hysterectomy: A clinical-pathologic analysis of a Gynecologic OncologyGroup/Southwest Oncology Group/Radiation Therapy Oncology Group trial. Gynecol Oncol 96: 721-728, 2005.

8. Vandecaveye V, Dresen R and De Keyzer F: Novel imaging techniques in gynaecological cancer. Curr Opin Oncol 29: 335-342, 2017.

9. Hanprasertpong J, Jiamset I, Geater A, Rattanaburi A and Thannil S: Clinical aspect and prognostic factors for survival in patients with recurrent cervical cancer after radical hysterectomy. Oncol Res Treat 39: 704-711, 2016.

10. Brooks RA, Rader JS, Dehdashti F, Mutch DG, Powell MA, Thaker PH, Siegel BA and Grigsby PW: Surveillance FDG-PET detection of asymptomatic recurrences in patients with cervical cancer. Gynecol Oncol 112: 104-109, 2009.

11. Wang J, Wang T, Yang YY, et al: Analysis of recurrence and metastasis after radical treatment of cervical cancer. Modern Oncol 21 (Suppl): S120-S122, 2013.

12. Elit L, Fyles AW, Devries MC, Oliver TK and Fung-Kee-Fung M; Gynecology Cancer Disease Site Group: Follow-up for women after treatment for cervical cancer: A systematic review. Gynecol Oncol 114: 528-535, 2009.

13. Landoni F, Maneo A, Colombo A, Placa F, Milani R, Perego P, Favini G, Ferri L and Mangioni C: Randomised study of radical surgery versus radiotherapy for stage Ib-IIa cervical cancer. Lancet 350: 535-540, 1997.

14. Xiaohong Y, Wei C, Huicheng X, et al: Observation of MRI in female pelvic floor. J Local Surg 21: 252-254, 2012.

15. Zhou XH, Ouyang SX and Xiao PZ: Applied anatomy and clinical significance of uterosacral ligament. J Nanhua Univ 35: 175-179, 2007.

16. Kong TW, Chang SJ, Piao X, Paek J, Lee Y, Lee EJ, Chun M and Ryu HS: Patterns of recurrence and survival after abdominal versus laparoscopic/robotic radical hysterectomy in patients with early cervical cancer. J Obstet Gynaecol Res 42: 77-86, 2016.

17. Ungár L, Pálfalvi L, Tarnai L, Nechushkina V, Lintner B and Novák Z; FACOG Committee on Practice Bulletins: Surgical treatment of stage IB cervical cancer. Int J Gynecol Cancer 22: 1597-1603, 2012.

18. Höckel M: Do we need a new classification for radical hysterectomy? Insights in surgical anatomy and local tumor spread from human embryology. Gynecol Oncol 107 (1 Suppl 1): S106-S112, 2007.

19. Höckel M, Horn LC, Manthey N, Braumann UD, Wolf U, Teichmann G, Frauenschläger K, Dornhöfer N and Einenkel J: Resection of the embryologically defined uterovaginal (Müllerian) compartment and pelvic control in patients with cervical cancer: A prospective analysis. Lancet Oncol 10: 683-692, 2009.

This work is licensed under a Creative Commons Attribution-NonCommercial-NoDerivatives 4.0 International (CC BY-NC-ND 4.0) License. 